\title{
Pulmonary rehabilitation and cardiovascular risk in COPD: a systematic review
}

\author{
Yousef S. Aldabayan", Ahmed M. Alrajeh, Arthur Lemson and John R. Hurst
}

\begin{abstract}
Introduction: Pulmonary Rehabilitation (PR) is an effective intervention in COPD however the value of PR in reducing cardiovascular risk in COPD (measured by aortic pulse wave velocity, aPWV) is unclear and there is no existing systematic review.

Objectives: To conduct a systematic review examining whether PR results in alteration of CV risk in COPD (as measured by aPW).

Methods: An electronic systematic search concordant with PRISMA guidelines was conducted. The search was complete to the 27th of May 2017. Six databases were examined: Embase, Medline, AMED, Web of Science, Cochrane clinical trials, and CINAHL.

Results: This study generated 767 initial matches, which were filtered using inclusion/exclusion criteria. Three studies (201 COPD participants) were included. Our analysis does not confirm that PR affects aPWV but studies were heterogeneous.

Conclusion: There is currently insufficient information on the effect of PR on reducing CV risk in COPD. Therefore controversy remains, with the possibility that there might be some subjects who benefit and others who might experience an increase in $\mathrm{CV}$ risk in response to PR. These results will be of value to those interested in gaining a better understanding of the benefits of PR on CV risk in COPD.
\end{abstract}

Keywords: Pulmonary rehabilitation, Cardiovascular, COPD, Arterial stiffness

\section{Introduction}

According to the World Health Organisation (WHO) [1], chronic obstructive pulmonary disease (COPD) is a leading cause of death. In part, the elevated mortality in COPD derives from an enhanced risk of cardiovascular disease (CVD) [2]. Without mitigation, COPD will be the third most common cause of death by 2030 [1].

COPD is defined as "a common, preventable and treatable disease that is characterized by persistent respiratory symptoms and airflow limitation that is due to airway and/or alveolar abnormalities usually caused by significant exposure to noxious particles or gases" [3]. Arterial stiffness is a gold-standard predictor of individual cardiovascular risk [4], and a biomarker of elevated cardiovascular risk in COPD patients [5], as it is in healthy populations. It can be measured using aortic pulse wave

\footnotetext{
* Correspondence: yousef.aldabayan.15@ucl.ac.uk

UCL Respiratory, Royal Free Campus, University College London, London NW3 2QG, UK
}

(c) The Author(s). 2017 Open Access This article is distributed under the terms of the Creative Commons Attribution 4.0 International License (http://creativecommons.org/licenses/by/4.0/), which permits unrestricted use, distribution, and reproduction in any medium, provided you give appropriate credit to the original author(s) and the source, provide a link to the Creative Commons license, and indicate if changes were made. The Creative Commons Public Domain Dedication waiver (http://creativecommons.org/publicdomain/zero/1.0/) applies to the data made available in this article, unless otherwise stated. creased arterial stiffness compared to controls $[7,8]$, and we have previously reported a relationship between the frequency of exacerbation and arterial stiffness [9].

Interventions to reduce cardiovascular risk in COPD patients are therefore of profound importance, yet existing evidence is not consistent and requires further investigation. Pulmonary Rehabilitation (PR) - a group exercise and education programme - is an evidence-based effective intervention in COPD to reduce symptoms, improve exercise performance and prevent exacerbations [10]. PR is defined by the European Respiratory Society (ERS) and American Thoracic Society (ATS) as "an evidence-based, multidisciplinary, and comprehensive intervention for patients with chronic respiratory diseases who are symptomatic and often have decreased daily life activities" [11]. Whilst exercise may reduce cardiovascular risk, the effect of exercise training and/or PR programme on cardiovascular risk in COPD remains controversial. The objective 
of this systematic review was to summarise and report the effect of PR on cardiovascular risk in COPD.

\section{Review \\ Method \\ Search strategy}

This systematic review is concordant with the Preferred Reporting Items for Systematic Reviews and MetaAnalyses (PRISMA) guidelines [12]. The search was complete to the 27th of May 2017. The search was performed using six electronic databases (Excerpta Medica dataBASE (Embase), MEDLARS Online (Medline), Allied and Complementary Medicine Database (AMED), Web of Science database, Cochrane clinical trials database, and Cumulative Index of Nursing and Allied Health Literature (CINAHL)).

All relevant titles and abstracts were read to evaluate eligibility based on our inclusion criteria. After reading the full texts, the first author eliminated non-relevant articles where potentially relevant articles were reviewed by the other authors to confirm eligibility.

\section{Inclusion and exclusion criteria}

The included studies met the following criteria:

1) Studies of patients with spirometrically-confirmed COPD.

2) Patients enrolled on a PR or an exercise training program.

3) Patients had cardiovascular risk assessment measured using aPWV before and after the programme to assess change in CV risk.

We excluded books, systematic reviews, non-English manuscripts, conference abstracts with no full-text and non-full text articles.

The primary outcome was change in cardiovascular risk measured using arterial stiffness in response to pulmonary rehabilitation or an exercise-training programme.

\section{Results}

Our search strategy (available in the Additional file 1), identified 767 articles, and one additional article was identified by screening reference lists. Of these, only three met all our inclusion and exclusion criteria (Fig. 1). Of the three included studies, two investigated COPD patients who had been enrolled on a PR programme, and one reported outcomes from patients enrolled on an exercise endurance training programme. In summary, with regard to the effect of PR on CV risk in COPD, we found one larger negative study [7] and two smaller positive studies $[5,6]$.

A detailed description of the three studies is given in Table 1. The sample size and duration of the included studies varied: 4 weeks, 7 weeks and two years. The sample size ranged from 17 to 330 participants.

\section{Quality assessment}

Among the three included studies, two were cohort studies and one was a randomized clinical trial. To assess the quality and the risk of bias of each article, the Newcastle-Ottawa scale (modified) [13] was used for cohort studies as indicated in Table 2 and the Cochrane risk of bias tool (modified) [14] was used for the randomised clinical trial as indicated in Table 3.

\section{Discussion}

This systematic review was conducted to evaluate the effects of exercise and/or pulmonary rehabilitation (PR) on cardiovascular risk in COPD as assessed by arterial stiffness. As reported in Table 1, there is one (larger) negative, and two (smaller) positive studies. Controversy remains over the effects of PR on cardiovascular risk. Studies were too heterogeneous to permit meta-analysis. This is the first systematic review to address the important question of whether PR affects cardiovascular risk in COPD.

Vivodtzev conducted a study in 2010 to investigate whether exercise training decreased arterial stiffness in patients with COPD. The randomized trial of aerobic endurance training was run for four weeks in 17 stable patients with moderate to severe COPD free from diabetes and respiratory exacerbation within the preceding three months. The participants were divided into two groups; a trained group $(n=10)$ and an untrained group $(n=7)$. Arterial stiffness was measured blindly by recording the carotid-radial pulse wave velocity (c-r PWV). The baseline measurements of c-r PWV were similar in both groups. After four weeks of an aerobic endurance training programme, c-r PWV readings were stable in the untrained group and significantly reduced in the trained group (from $10.3 \pm 0.7$ to $9.2 \pm 0.8 \mathrm{~m} / \mathrm{s}$, $P=0.001)$. c-r PWV reduction correlated with improvements in walking distance $(r=0.49)$, systolic blood pressure $(r=0.79)$, muscle endurance $(r=0.48)$, and fasting glucose $(r=0.59)$ in all patients $(P=<0.05)$, and with changes in maximal heart rate and oxygen consumption $(r=0.70, P=0.02)$ in trained patients. The authors concluded that arterial stiffness appears to be improved following exercise in stable patients with COPD.

Gale conducted a study in 2011 to investigate if pulmonary rehabilitation affected cardiovascular risk factors in patients with COPD. The study was run for seven weeks in the context of a multidisciplinary pulmonary rehabilitation programme on a total of 32 stable COPD participants who were free from ischemic heart disease, cardiac failure, diabetes mellitus or malignancy. These 32 participants were compared with 20 healthy controls at the baseline assessment. In all subjects, aortic pulse 


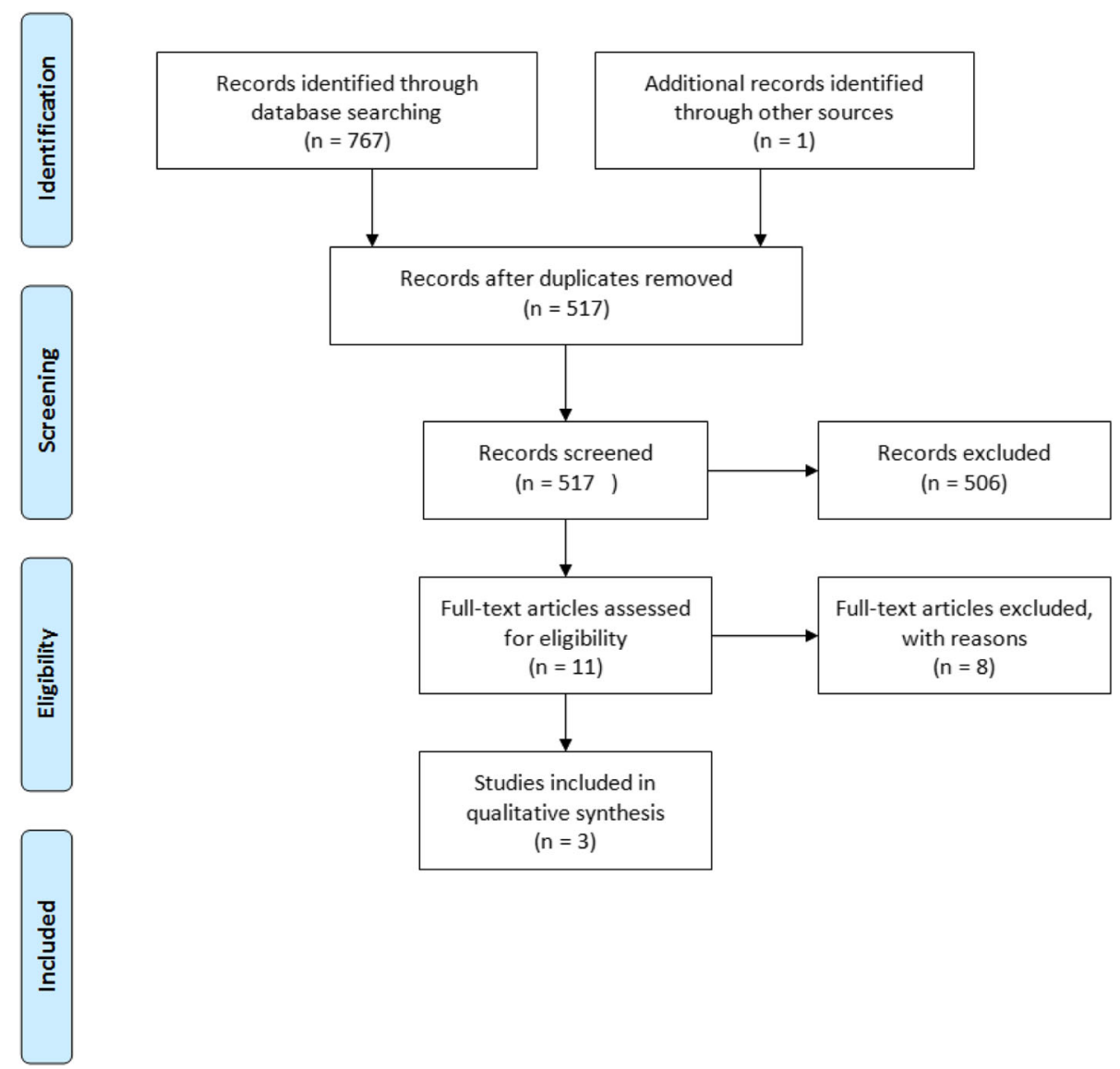

Fig. 1 PRISMA Flow Diagram

wave velocity was measured before the programme and repeated for those who completed PR. On the initial measurements of aortic stiffness, aPWV was increased in patients compared to the control group $(p<0.05)$. After seven weeks of the multidisciplinary pulmonary rehabilitation programme, 22 patients completed the programme. aPWV was re-measured for those who completed the programme, and was significantly reduced in comparison to the baseline measurement (from $9.8 \pm 3.0 \mathrm{~m} / \mathrm{s}$ to $9.3 \pm 2.7 \mathrm{~m} / \mathrm{s}, p=\leq 0.05$ ). Gale concluded that cardiovascular risk measured by aortic stiffness was significantly improved following multidisciplinary pulmonary rehabilitation in patients with COPD.

Vanfleteren conducted a study in 2014 that aimed to confirm increased arterial stiffness in COPD, evaluate its relationship with systemic inflammation and, most importantly, to examine if the increased arterial stiffness was influenced by PR. The study was run for two years on a total of 168 healthy control participants who had normal pulmonary function tests and 162 COPD patients. Among the COPD group, only 129 patients completed the PR programme and were re-assessed. It was reported that arterial stiffness was increased in COPD patients at baseline measurement compared to controls. Furthermore, it was reported that systemic inflammatory markers were not related to increased arterial stiffness in COPD. Overall, there was no change in aPWV measurement after PR $(10.7 \pm 2.7$ versus $10.9 \pm 2.5 \mathrm{~ms}, p=0.339)$. The Authors concluded that changes in arterial stiffness in COPD were not related to markers of systemic inflammation and not influenced by pulmonary rehabilitation.

We noted heterogeneity across the included studies regarding exercise intensity and programme duration (and such heterogeneity prevented meta-analysis). In the Gale study [6], arterial stiffness significantly improved after PR. Additionally, there was a significant reduction in central mean arterial pressure $(103 \pm 14$ to $95 \pm 14 \mathrm{mmHg}$; $p=<0.001)$ which may account for the reduction in aPWV. The larger negative study by Vanfleteren [7] was well designed consisting of 40 sessions, twice as many as Gale [6]. There was no effect on reduction of aPWV or on mean arterial pressure. The Vivodtzev study did not include an education component [5].

The available data on the ability of PR to reduce cardiovascular risk in COPD therefore remains contradictory. Importantly, we noted that $\mathrm{CV}$ responses to 


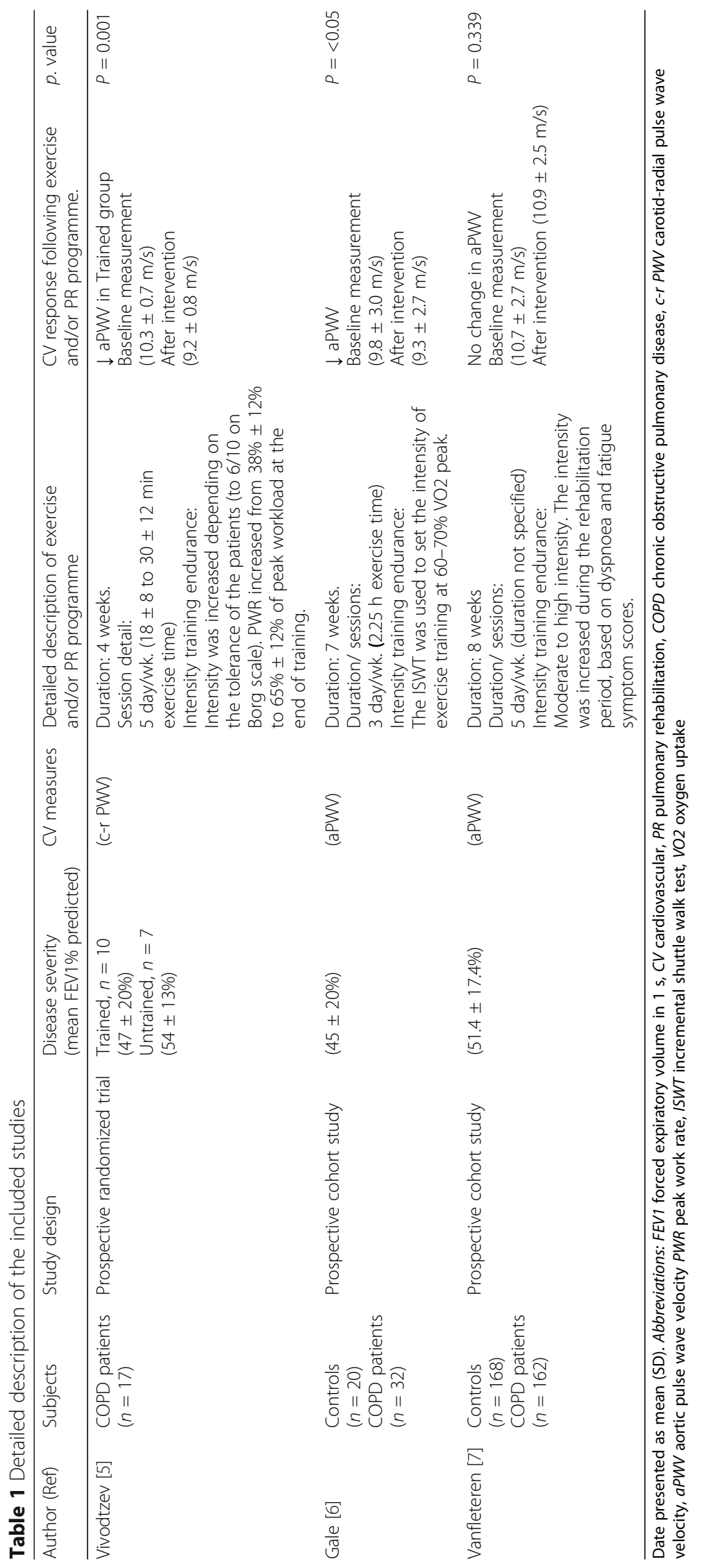


Table 2 Risk of bias of the included cohort studies

\begin{tabular}{|c|c|c|c|c|c|c|c|}
\hline First author & $\begin{array}{l}\text { Population } \\
\text { representative }\end{array}$ & $\begin{array}{l}\text { Sample size } \\
\text { adequate }\end{array}$ & Confounders & Statistical analysis & Missing data & $\begin{array}{l}\text { Methodology of } \\
\text { the outcome }\end{array}$ & $\begin{array}{l}\text { Objective } \\
\text { assessment }\end{array}$ \\
\hline Gale 2011 [6] & 3 & 2 & 3 & 3 & 3 & 3 & 3 \\
\hline Vanfleteren 2014 [7] & 3 & 3 & 3 & 3 & 3 & 3 & 3 \\
\hline
\end{tabular}

$0=$ Definitely no (therefore high risk of bias), $1=$ Mostly no, $2=$ Mostly yes, 3 = Definitely yes (therefore low risk of bias)

PR appeared highly heterogeneous in the Vanfleteren paper [7]. This raises the important hypothesis that some patients may benefit and that some patients may be at increased risk from PR in COPD (Fig. 2, derived from the original paper [7]. This may be clinically important.

Cardiovascular co-morbidity is common in COPD. Chen conducted a systemic review and meta-analysis aiming to quantify the magnitude of the association between COPD and increased prevalence of cardiovascular risk-factors and co-morbidity [15]. Twenty seven observational studies were included in the meta-analysis. The pooled odds of cardiovascular disease was higher in subjects with COPD compared to people without (metaodds ratio OR 2.46, 95\% CI 2,02-3.00; $p<0.0001$ ). This comprised a two to five-fold higher risk of major cardiovascular disease sub-types (ischemic heart disease, cardiac dysrhythmia, heart failure, diseases of the pulmonary circulation and arterial diseases), and a one-third increased risk of hypertension and diabetes. No significant increased risk of cerebrovascular disease, dyslipidaemia, and obesity was detected in patients with COPD.

The papers examined in this systematic review included information on baseline cardiovascular risk. In the Vanfleteren paper, among 162 COPD patients there was no statically significant difference in aPWV after PR $(p=0.339)$ despite the prevalence of recorded cardiac comorbidities including myocardial infraction $(n=16$ [9.9\%]), heart failure $(n=5[3.1 \%])$, peripheral arterial diseases $(n=30$ [18.5\%]), cerebrovascular disease $(n=14[8.6 \%])$, diabetes $(n=7[4.3 \%])$ or any other cardiovascular diseases $(n=50$ [30.9\%]). Furthermore, even after subdividing the patients into subgroups based on cardiovascular risk factors including smoking (ever smoked), hypertension, hyperlipidemia, age and obesity there was no notable difference in response $(p>0.05)$. In the positive studies, Vivodtzev reported that none of the participants had a history of ischemic heart disease or stroke. They reported that the significant reduction in PWV correlated with systolic BP $(r=0.79)$ and fasting glucose $(r=0.59)$. In the Gale study, none of the participants had reported cardiovascular disease. However, PR did improve some cardiovascular risk factors, including both blood pressure and cholesterol $(p=<0.05)$.

None of the three included studies in this review reported data on subsequent risk of hospital admission or mortality following the exercise programme. Puhan conducted a systematic review aiming to compare the effect of PR after COPD exacerbation in reducing hospital admission and mortality [16]. Twenty RCTs (1477 participants) were included in this review. Eight studies including 810 participants contributed data on hospital readmission indicating that $\mathrm{PR}$ reduced hospital readmission (pooled odds ratio (OR) 0.44 , CI 0.21 to 0.91 ) but the results were heterogeneous (77\%). Six studies contributed data on mortality. The duration of follow-up period ranged from 3 to 48 months with one study showing reduced mortality and four no effect. The quality of evidence was low. None of the trials considered mortality as a primary outcome and the analysis did not show a statistically significant influence of PR on mortality $(\mathrm{OR}=0.68, \mathrm{CI} 0.28$ to 1.67$)$ with significant heterogeneity reported across the studies.

It can be seen from the analysis above that CVD and CVRs are very prevalence among COPD patients, which might be expected to proportionally influence the aPWV. Therefore, the variety of the included patients in terms of CVDs and CVRs might also count to explain the current results' heterogeneity of this systematic review.

The available data on the ability of PR to reduce cardiovascular risk assessed by $\mathrm{APWV}$ in COPD therefore remains contradictory. Interestingly, Oliveira performed a systemic review with the aim of examining the effect of exercise training on arterial stiffness in Coronary Artery Disease (CAD) patients assessed by measuring arterial stiffness or related indexes (eg. indexes of wave

Table 3 Risk of bias in the included randomised trial

\begin{tabular}{|c|c|c|c|c|c|c|c|}
\hline First author & $\begin{array}{l}\text { Random sequence } \\
\text { generation }\end{array}$ & $\begin{array}{l}\text { Allocation } \\
\text { concealment }\end{array}$ & $\begin{array}{l}\text { Blinding of participants } \\
\text { and personnel }\end{array}$ & $\begin{array}{l}\text { Blinding of outcome } \\
\text { assessment }\end{array}$ & $\begin{array}{l}\text { Incomplete } \\
\text { outcome data }\end{array}$ & $\begin{array}{l}\text { Selective } \\
\text { reporting }\end{array}$ & $\begin{array}{l}\text { Other sources } \\
\text { of bias }\end{array}$ \\
\hline Vivodtzev 2010 [5] & Unclear & Unclear & Unclear & Low & Low & Unclear & Low \\
\hline
\end{tabular}

Low: Low risk of bias, High: High risk of bias, Unclear: Unclear risk of bias 


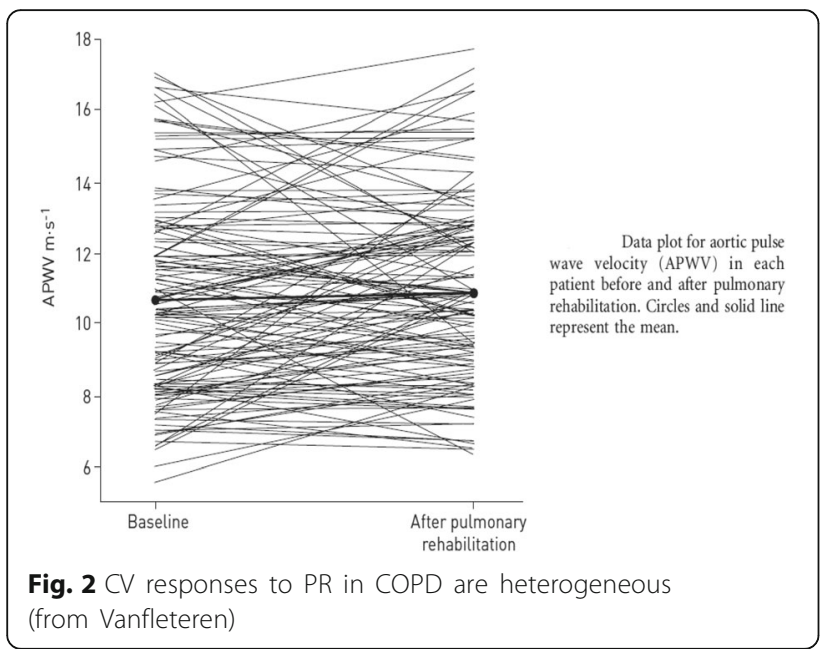

reflection or arterial compliance) [17]. Five studies were included in this review with a total of 271 participants varying in age (48-67 years) and exercise programme duration (6 to 20 weeks). Participants were diagnosed with CAD after an acute myocardial infarction, coronary artery bypass graft or coronary angioplasty. Overall, Oliveira found that exercise programs in CAD patients led to improved outcome measures. Specifically, there was a significant reduction in PWV despite use of different measurement methods including brachial-ankle PWV (reduction of $0.120 \mathrm{~m} / \mathrm{s})$, carotid-femoral PWV $(4.1 \mathrm{~m} / \mathrm{s}$ to $-0.7 \mathrm{~m} / \mathrm{s})$ and carotid-radial $\operatorname{PWV}(0.7 \mathrm{~m} / \mathrm{s})$. The authors reported a greater reduction in PWV in CAD patients who completed a 20 -week $(-1.0 \pm 0.3 \mathrm{~m} / \mathrm{s})$ versus a 12 -week training course $(-0.6 \pm 0.2 \mathrm{~m} / \mathrm{s})$.

In summary, we are unable to conclude whether there is or is not a significant change in aortic pulsewave velocity in response to PR in COPD. Therefore further work is required to definitively address this question and in particular whether there may be subgroups of patients who do and do not respond. There is an urgent unmet need for evidence-based standardised interventions that reduce the risk of cardiovascular events in patients with COPD. Conducting further studies considering confounding variables such as cardiovascular risk factors and disease, medication, exercise intensity and duration of the programme may better assess the benefits of $\mathrm{PR}$ programme on $\mathrm{CV}$ risk in COPD.

\section{Strength and limitations}

The strength of this work is that there is no previous systematic review on the effect of $\mathrm{PR}$ on $\mathrm{CV}$ risk in COPD measuring aPWV. A potential limitation of this report was that we excluded non-English studies.

\section{Conclusion}

We have systematically searched for and reported those studies that evaluated the effect of PR on CV outcomes in COPD. There is some existing data evaluating the effect of PR on cardiovascular risk in COPD, with one (larger and higher quality) negative study and two smaller positive ones. Study heterogeneity results in continuing controversy in the field. The negative study also reported inter-patient heterogeneity in responses that are unexplained, require further evaluation and may be clinically important. Results generated from this report will be of importance for researchers interested in assessing the benefits of PR on reducing CV risk in COPD.

\section{Additional file}

Additional file 1: Search Strategy. (DOC $146 \mathrm{~kb})$

\section{Abbreviations}

AMED: Allied and complementary medicine database; aPWV: Aortic pulse wave velocity; ATS: American thoracic society; BP: Blood pressure;

CINAHL: Cumulative index of nursing and allied health literature; COPD CV: Cardiovascular; c-r PWV: Carotid-radial pulse wave velocity;

CVD: Cardiovascular disease; CVR: Cardiovascular risk; Embase: Excerpta Medica dataBASE; ERS: European Respiratory Society; FEV1: Forced expiratory volume in 1 second; ISWT: Incremental shuttle walk test; MAP: Mean arterial pressure; PR: Pulmonary rehabilitation; PRISMA: Preferred reporting items for systematic reviews and meta-analyses; PWR: Peak work rate; UK: United Kingdome; VO2: Oxygen uptake; WHO: World Health Organisation

\section{Acknowledgements}

We thank Angela Young, Medical Librarian at Royal Free London NHS Foundation Trust, UK for her assistance and support in refining the search strategy. We thank King Faisal University represented by the Saudi Arabian Cultural Bureau in London for funding Yousef Aldabayan and Ahmed Alrajeh.

\section{Funding}

This work is supported by King Faisal University through the Saudi Arabian Cultural Bureau in London.

\section{Availability of data and materials}

Please contact the corresponding author for data requests.

\section{Authors' contributions}

YA designed the study and carried out the initial search and data extraction, and drafted the manuscript. AA and $\mathrm{JH}$ participated in the design of the study with YA. AL assisted with contributions on PR and cardiovascular risk in coronary artery disease. All authors reviewed and approved the final manuscript.

Ethics approval and consent to participates

This is a systematic review, ethics approval is not applicable.

Consent for publication

This is a systematic review, consent is not applicable.

Competing interests

The authors declare that they have no competing interests.

\section{Publisher's Note}

Springer Nature remains neutral with regard to jurisdictional claims in published maps and institutional affiliations. 
Received: 18 April 2017 Accepted: 23 June 2017

\section{Published online: 06 July 2017}

\section{References}

1. Prins KW, Markowitz J, Pritzker M, et al. Use of PAH-specific therapy in world health organization group iii pulmonary hypertension: a systematic review and meta-analysis. J Heart Lung Transplant. 2016;1:S175.

2. Huiart L, Ernst P, Suissa S. Cardiovascular morbidity and mortality in COPD. Chest. 2005;128(4):2640-6.

3. From the Global Strategy for the Diagnosis MaPoC, Global Initiative for Chronic Obstructive Lung Disease (GOLD) 2017. Available from: http:// goldcopd.org. Secondary. Accessed 3 July 2017.

4. Hickson SS, Butlin M, Broad J, et al. Validity and repeatability of the Vicorder apparatus: a comparison with the SphygmoCor device. Hypertens Res. 2009; 32(12):1079-85.

5. Vivodtzev I, Minet C, Wuyam B, et al. Significant improvement in arterial stiffness after endurance training in patients with COPD. Chest. 2010;137(3): 585-92.

6. Gale NS, Duckers JM, Enright S, et al. Does pulmonary rehabilitation address cardiovascular risk factors in patients with COPD? BMC Pulm Med. 2011;11:20

7. Vanfleteren L, Spruit MA, Groenen MTJ, et al. Arterial stiffness in patients with COPD: the role of systemic inflammation and the effects of pulmonary rehabilitation. Eur Respir J. 2014;43(5):1306-15.

8. Cinarka H, Kayhan S, Gumus A, et al. Arterial stiffness measured via carotid femoral pulse wave velocity is associated with disease severity in COPD. Respir Care. 2014:59(2):274-80.

9. Patel AR, Kowlessar BS, Donaldson GC, et al. Cardiovascular risk, myocardial injury, and exacerbations of chronic obstructive pulmonary disease. Am J Respir Crit Care Med. 2013;188(9):1091-9.

10. McCarthy B, Casey D, Devane D, et al. Pulmonary rehabilitation for chronic obstructive pulmonary disease. Cochrane Database Syst Rev. 2015;2: CD003793.

11. Gibson GJ, Loddenkemper R, Sibille Y, et al. The European Lung White Book: Respiratory Health and Disease in Europe: European respiratory Society, 2013.

12. Moher D, Liberati A, Tetzlaff J, et al. Preferred reporting items for systematic reviews and meta-analyses: the PRISMA statement. Open Medicine: A Peerreviewed, Independent, Open-access Journal. 2009:3(3):e123-30.

13. Adapted version of a modified Newcastle-Ottawa Scale for single use in specific context. Secondary Adapted version of a modified NewcastleOttawa Scale for single use in specific context. http://www.biomedcentral. com/content/supplementary/2046-4053-3-45-S2.pdf. Accessed 3 July 2017.

14. Cochrane Risk Of Bias Tool (Modified) For Quality Assessment Of Randomized Controlled Trials. Secondary Cochrane Risk Of Bias Tool (Modified) For Quality Assessment Of Randomized Controlled Trials. http:// www.tc.umn.edu/ msrg/caseCATdoc/rct.crit.pdf. Accessed 3 July 2017

15. Chen W, Thomas J, Sadatsafavi M, et al. Risk of cardiovascular comorbidity in patients with chronic obstructive pulmonary disease: a systematic review and meta-analysis. Lancet Respir Med. 2015:3(8):631-9.

16. Puhan MA, Gimeno-Santos E, Cates CJ, et al. Pulmonary rehabilitation following exacerbations of chronic obstructive pulmonary disease. Cochrane Database Syst Rev. 2016;12:CD005305.

17. Oliveira NL, Ribeiro F, Alves AJ, et al. The effects of exercise training on arterial stiffness in coronary artery disease patients: a state-of-the-art review. Clin Physiol Funct Imaging. 2014:34(4):254-62

\section{Submit your next manuscript to BioMed Central and we will help you at every step:}

- We accept pre-submission inquiries

- Our selector tool helps you to find the most relevant journal

- We provide round the clock customer support

- Convenient online submission

- Thorough peer review

- Inclusion in PubMed and all major indexing services

- Maximum visibility for your research

Submit your manuscript at www.biomedcentral.com/submit 